\title{
Selective Fluorescence Quenching to Discriminate Between Alternant and Non- alternant Polycyclic Aromatic Hydrocarbons: Acephenanthrylene Derivatives as Exceptions to the Nitromethane Quenching Rule
}

\author{
Sheryl A. Tucker, Jason M. Griffin and William E. Acree, Jr.* \\ Department of Chemistry. University of North Texas, Denton, TX 76203-5068, \\ USA
}

Patrick P. J. Mulder, Johan Lugtenburg and Jan Cornelisse

Gorlaeus Laboratories, Leiden University, P.O. Box 9502, 2300 RA Leiden,

The Netherlands

\begin{abstract}
Nitromethane selectively quenches the fluorescence emission of alternant polycyclic aromatic hydrocarbons (PAHs) via an electron transfer mechanism. Emission intensities of nonalternant PAHs, for the most part, are unaffected. Results of previous measurements show that nitromethane does quench the fluorescence emission of the nine acenaphthylene solutes studied, which is completely contrary to what would be expected based on the fact that all nine solutes are nonalternant PAHs. To investigate this phenomenon further, the fluorescence behaviour of 1-, 3-, 4-, 5-, 6-, 7-, 8-, 9- and 10methylacephenanthrylene dissolved in neat acetonitrile, acetonitrile-ethyl acetate and acetonitrile-toluene solvents was measured at different nitromethane concentrations. Experimental results support the notion that the double bond in the five-membered ring is fixed; therefore, the solutes act as alternant rather than non-alternant PAHs.
\end{abstract}

Keywords: Fluorescence; quenching; polycyclic aromatic hydrocarbons; acephenanthrylenes; alternant and non-alternant

\section{Introduction}

The identification and determination of polycyclic aromatic hydrocarbons (PAHs) in unknown mixtures require accurate fluorescence emission intensity measurements and the availability of a large spectral data file for comparing the spectrum of an unknown against those of PAH standards. Mixtures of environmental and/or industrial importance rarely contain a single component. Most mixtures commonly encountered contain several isomeric pairs or structurally similar PAHs, which emit in approximately the same spectral regions. Kalman filtering and Gaussian or other curve-fitting techniques, ${ }^{1-6}$ alone or in combination with phase-resolved ${ }^{7-9}$ or synchronous scanning ${ }^{10-12}$ fluorescence spectrometry, theoretically allow the uncoupling of overlapped spectra. Such methods become less reliable, however, as the number of mixture components increases. High-performance liquid chromatographic (HPLC) separation prior to fluorimetric analysis affords a viable alternative, but again the method is extremely time consuming whenever large numbers of isomeric compounds are present. Blümer and Zander ${ }^{13}$ recommended that nitromethane and/or nitrobenzene could be added to a water-acetonitrile $(20+80 \mathrm{v} / \mathrm{v})$ binary mobile

* To whom correspondence should be addressed. phase to suppress selectively fluorescence signals of alternant PAHs. Emission intensities of non-alternant PAHs would remain unchanged. In separations where an alternant and non-alternant $\mathrm{PAH}$ co-elute, as is often the case, the detector would respond to only the non-alternant PAH.

Published studies ${ }^{14-21}$ involving over 96 PAHs have identified dibenzo[ $h i, w x]$ heptacene, benzo[ $k]$ fluoranthene and naphtho[ $2,3 b]$ fluoranthene as among the few exceptions to the so-called nitromethane selective quenching rule in the PAH6 benzenoid, fluorenoid, fluoranthenoid and 'methylenebridged' cyclopenta-PAH subclasses. Also, the fluorescence emission of all nine non-alternant acenaphthylene derivatives 22 included in the studies were quenched by nitrometh ane, which is completely contrary to what would be expected based on the selective quenching rule. Their peculiar behaviour was attributed to the fixed double bond in the cyclopenta ring, which is unlike previously examined non-alternant $\mathrm{PAH}$ subclasses, e.g., fluorenoids and fluoranthenoids. To investigate this phenomenon further, the fluorescence behaviour of 1-, 3-, 4-, 5-, 6-, 7-, 8-, 9- and 10-methylacephenanthrylene (MAP) dissolved in neat acetonitrile, acetonitrile-ethyl acetate and acetonitrile-toluene solvents was measured at different nitromethane concentrations. The molecular structures of the nine non-alternant acephenanthrylene derivatives are shown in Fig. 1.23 Also included is a discussion of the fluorescence behaviour of the acephenanthrylene derivatives in organic non-electrolyte solvents of various polarity.

\section{Experimental}

The acephenanthrylene solutes were synthesized and purified by procedures described in the literature. ${ }^{24}$ Stock standard solutions were prepared by dissolving solutes in dichloromethane. Small aliquots of the stock standard solutions were transferred into the test-tubes, allowed to evaporate and diluted with the solvent of interest. The final solute concentrations were sufficiently dilute to minimize inner-filtering artifacts. Solvents were of HPLC, spectral or analyticalreagent grade, purchased from either Aldrich or Fisher Scientific, and the resulting solutions were optically dilute (absorbance $\mathrm{cm}^{-1}<0.01$ ) at all wavelengths, except for the nitromethane quenching studies.

Absorption spectra were recorded on a Bausch and Lomb Spectronic 2000 and a Hewlett-Packard 8450 A photodiodearray spectrophotometer in the usual manner. The fluorescence spectra were measured on a Shimadzu RF-5000U spectrofluorimeter with the detector set at high sensitivity. 
Solutions were excited at 304 (1-MAP), 300 (3-MAP), 300 (4MAP), 315 (5-MAP), 300 (6-MAP), 304 (7-MAP), 300 (8MAP), 300 (9-MAP) and $306 \mathrm{~nm}$ (10-MAP). Fluorescence data were accumulated in a $1 \mathrm{~cm}^{2}$ quartz cuvette at $19{ }^{\circ} \mathrm{C}$ (room temperature) with excitation and emission slit width settings of 15 and $3 \mathrm{~nm}$, respectively. The fluorescence spectra of the acephenanthrylene derivatives, depicted in Figs. 2 and 3 , represent a single scan which was then solvent blank corrected and verified by repetitive measurements.

Emission intensities associated with the quenching study were corrected for primary inner-filtering artifacts arising from the absorption of excitation radiation according to the following expression: $25-27$

$$
f_{\text {prim }}=\frac{F \text { corr }}{F_{\text {obs }}}=\frac{2.303 A(y-x)}{10^{-A x}-10^{-A y}}
$$

which differs slightly from the approximate form ${ }^{28}$

$$
f_{\text {prim }} \approx 10^{0.5 \mathrm{~A}}
$$

where $F^{\mathrm{corr}}$ and $F^{\mathrm{obs}}$ refer to the corrected and observed fluorescence emission signal, respectively, $A$ is the absorbance per centimetre of pathlength at the excitation wavelength and $x$ and $y$ denote distances from the boundaries of the interrogation zone to the excitation plane. Several of the PAHs have excitation wavelengths in the $300-320 \mathrm{~nm}$ region and a few drops of nitromethane gave solutions having appreciable absorbances. Computational procedures and interrogation zone dimensions are discussed in greater detail elsewhere. ${ }^{14,19,20,29,30}$ Every effort was made to work at solution absorbances of $A \mathrm{~cm}^{-1} \leqslant 0.95\left(f_{\text {prim }} \leqslant 3.00\right)$, where the inner-filtering correction equation is valid. Secondary inner-filtering corrections were not necessary as nitromethane is 'optically transparent' in most of these PAH emission ranges.

\section{Results and Discussion}

Representative fluorescence emission spectra of 3-MAP and 4-MAP dissolved in hexadecane, butyl acetate, dichloromethane and dimethyl sulfoxide (DMSO) are depicted in Figs. 2 and 3. These four non-electrolyte solvents were judiciously selected so as to encompass the entire range of solvent polarity, from the non-polar hexadecane hydrocarbon through the moderately polar butyl acetate and dichloromethane to the very polar DMSO, which is the most polar solvent considered. Examination of the spectral data reveals that none of the acephenanthrylene derivatives can be classified as solvent polarity probe molecules. However, 1-, 4-, 6-, 7-, 8-, 9- and 10-MAP did show non-systematic variations of emission intensity ratios with solvent polarity. For example, the calculated emission intensities for 6- and 9-MAP, (I 358/ III $\sim 396 \mathrm{~nm}$ for both solutes) were 6-MAP $=4.11$ and 9-MAP $=4.76$ in hexadecane, 6 - MAP $=3.36$ and 9-MAP $=3.53$ in butyl acetate, $6-\mathrm{MAP}=2.86$ and $9-\mathrm{MAP}=2.66$ in methanol and 6-MAP $=3.39$ and 9-MAP $=2.87$ in DMSO. Solvent polarity probe ratios for methanol and butyl acetate (both moderately polar) for most of the seven solutes are consistently lower than the ratios obtained in DMSO, despite the fact that DMSO is the most polar solvent examined. Lower than expected emission intensity ratios in methanol and butyl acetate prevented the five MAPs from being classified as solvent polarity probe molecules. Estimated uncertainties in the measured intensity ratios are believed to be on the order of \pm 0.10 (or less) on the basis of replicate measurements.

Fig. 3 also shows excimer formation in the 4-MAP fluorescence emission spectra. The low quantum yields of some solutes in this class of PAHs caused difficulties in obtaining a fluorescence emission spectra. The concentrations of 4-, 5- and 9-MAP had to be raised so that sufficiently intense fluorescence emission spectra could be obtained for the nitromethane selective quenching studies which caused excimer formation. Concentrational studies were performed
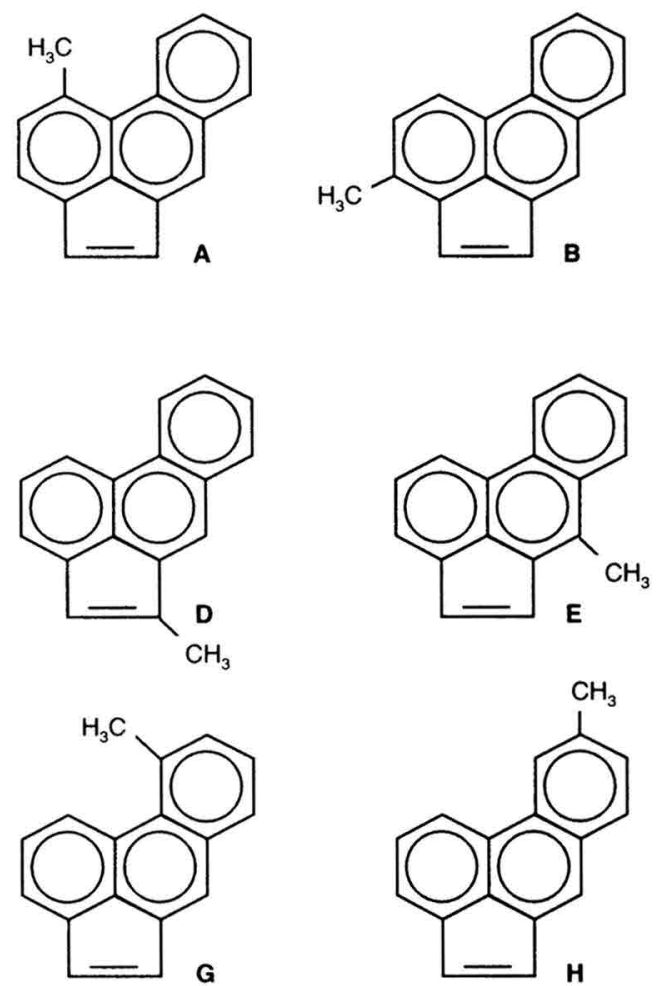<smiles></smiles><smiles>Cc1cccc2c1cc1c3c(cccc32)C=C1</smiles>

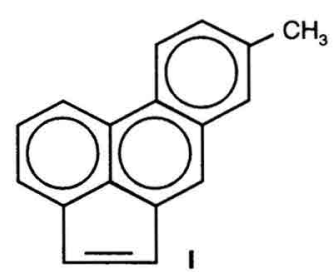

Mos. Molicular structures of the acephenanthrylene derivatives: A, 1-; B, 3-; C, 4-; D, 5-; E, 6-; F, 7-; G, 10-; H, 9-; and I, 8-methylaceahesantirylene. 
to verify that the red-shifted peak $(\sim 493 \mathrm{~nm}$ for 4-MAP and $\sim 505 \mathrm{~nm}$ for 5- and 9-MAP) seen in Fig. 3 was indeed excimer formation and not some other artifact.

The effect of nitromethane on all nine acephenanthrylene solutes dissolved in acetonitrile, acetonitrile-ethyl acetate $(60: 40 \mathrm{v} / \mathrm{v})$ and acetonitrile-toluene $(60: 40 \mathrm{v} / \mathrm{v})$ was also examined. In all cases one Pasteur pipette drop of nitromethane added to $3 \mathrm{ml}$ of solution decreased the fluorescence signal to about $10-20 \%$ of its original value after correction for primary inner filtering. Nitromethane quenched the fluorescence emission of all nine PAHs, examined. Their behaviour is identical with that of the nine non-alternant acenaphthylene derivatives examined earlier that had quenching behaviour indicative of alternant rather than non-alternant PAHs. ${ }^{22}$ The acenaphthylene and acephenanthrylene derivatives are the first subclasses of PAHs in which all solutes examined behave as exceptions to the selective quenching rule. Quenching behavioural differences between alternant and non-alternant

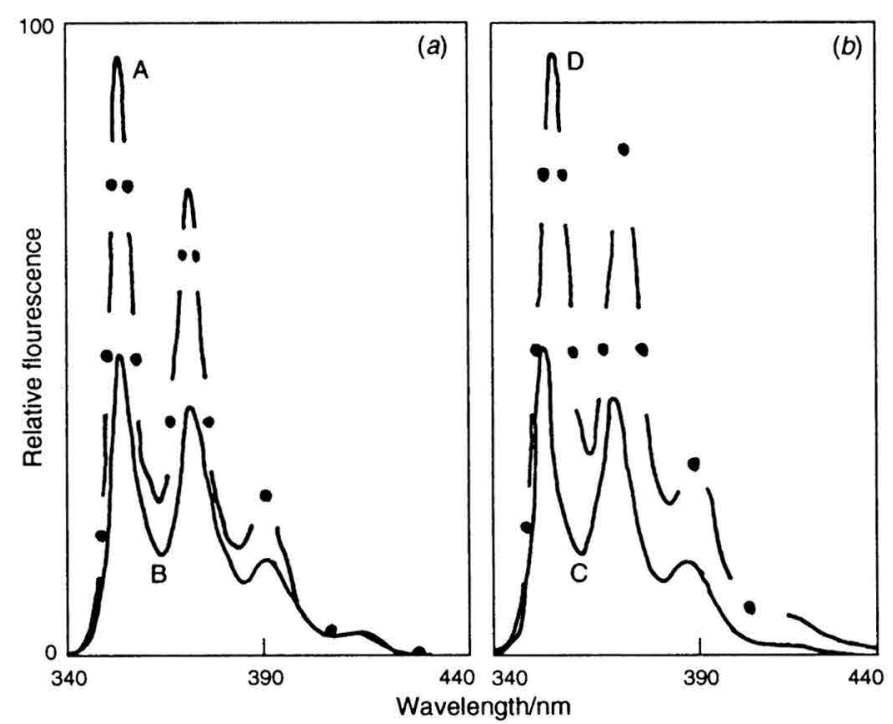

Fig. 2 Fluorescence emission spectra of 3-methylacephenanthrylene dissolved in: A. hexadecane; B, dichloromethane; C, butyl acetate; and $\mathrm{D}$, dimethyl sulfoxide. In butyl acetate emission bands occur at about $353,372,391$ and $413 \mathrm{~nm}$.

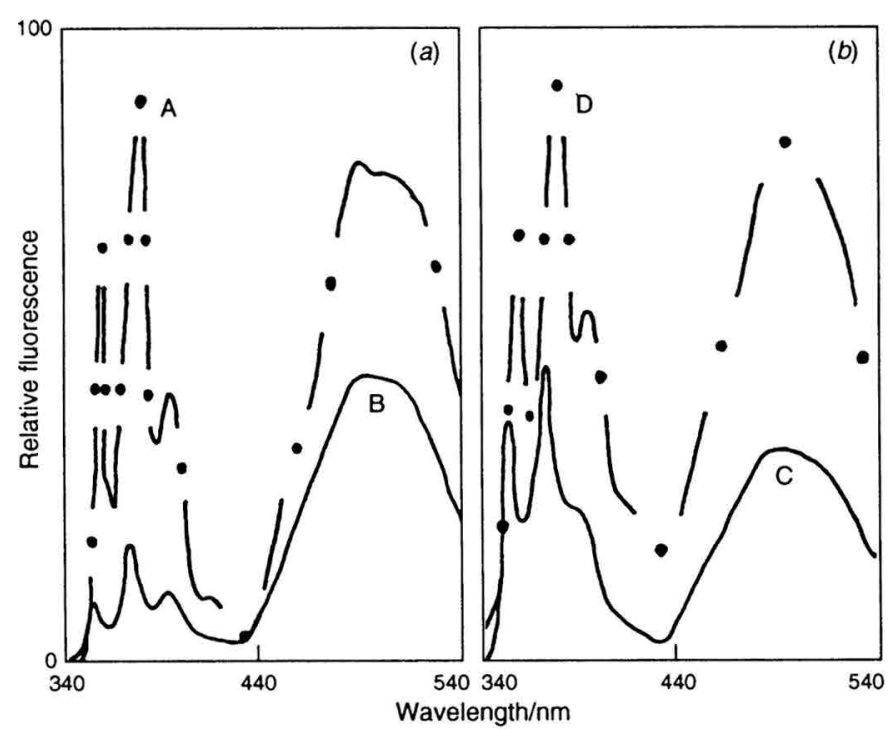

Fig. 3 Fluorescence emission spectra of 4-methylacephenanthrylene dissolved in: A, hexadecane; B, dichloromethane; C, butyl acetate; and $D$, dimethyl sulfoxide. In butyl acetate emission bands occur at about $354,372,391$ and $490 \mathrm{~nm}$.
PAHs have been rationalized ${ }^{18,31}$ in terms of processes originating from the vibrationally relaxed first electronic excited singlet state, ${ }^{1} \mathrm{PAH}^{*}$. Deactivation from the ${ }^{1} \mathrm{PAH}^{*}$ state is governed by the competititon between radiative and non-radiative processes. Rate constants of fluorescence decay, $k_{\text {fluores }}$, for PAH fluorophores are generally insensitive to molecular environment. Efficiencies of non-radiative proceses, on the other hand, depend to a large extent on external perturbations resulting from interactions involving $\mathrm{PAH}$ solutes with solvent/quenching molecules. Three possible mechanisms include reduction of fluorescence emission intensities through (1) intersystem crossing processes involving external 'heavy atom' quenchers:

$$
{ }^{1} \mathrm{PAH}^{*}+{ }^{1} \text { Quencher } \rightarrow{ }^{3} \mathrm{PAH}^{*}+{ }^{1} \text { Quencher }
$$

(2) intermolecular transfer (or partial transfer; $\delta+/ \delta-$ ) of a single electron to form free ions (or charge-transfer complex):

${ }^{1} \mathrm{PAH}^{*}+{ }^{1}$ Quencher $\rightleftharpoons\left(\mathrm{PAH}^{+/-} .\right.$. Quencher $\left.{ }^{-1+}\right) \rightarrow$ ${ }^{2} \mathrm{PAH}^{+1-}+{ }^{2} \mathrm{Quencher}^{-1+}$

and (3) intermolecular electronic energy transfer:

$$
{ }^{1} \mathrm{PAH}^{*}+{ }^{1} \text { Quencher } \rightarrow{ }^{1} \mathrm{PAH}+{ }^{3} \text { Quencher }{ }^{*}
$$

with the quenching agent promoted from a ground singlet state to an excited triplet state, requiring energy $\left({ }^{1} \mathrm{PAH}^{*}\right)>$ energy ( ${ }^{3}$ Quencher*).

Of the aforementioned mechanisms, only the second could discriminate between alternant and non-alternant PAHs. Breymann et al. ${ }^{18}$ attributed the selectivity of nitromethane to an electron/charge-transfer reaction. They argued that the reduction potentials of non-alternant PAHs are generally 0.4 $\mathrm{eV}$ more positive than those of alternant PAHs. Quantum mechanical computations show the highest occupied molecular orbital (HOMO) and lowest unoccupied molecular orbital (LUMO) energies of non-alternant PAHs to be lowered against those of alternant PAHs of equal HOMOLUMO separation. For the electron transfer reaction,

$$
{ }^{1} \mathrm{PAH}^{*}+{ }^{1} \text { Quencher } \rightarrow{ }^{2} \mathrm{PAH}^{+}+{ }^{2} \mathrm{Quencher}^{-}
$$

the change in free energy is ${ }^{18}$

$\Delta G \approx \operatorname{energy}\left({ }^{1} \mathrm{PAH}^{*} / 2 \mathrm{PAH}^{+}\right)-\operatorname{energy}\left({ }^{2} \mathrm{Quencher}^{-} /\right.$ 'Quencher $)+$ other term $(\mathrm{s}) \approx \operatorname{energy}\left({ }^{1} \mathrm{PAH} / 2 \mathrm{PAH}^{+}\right)-$

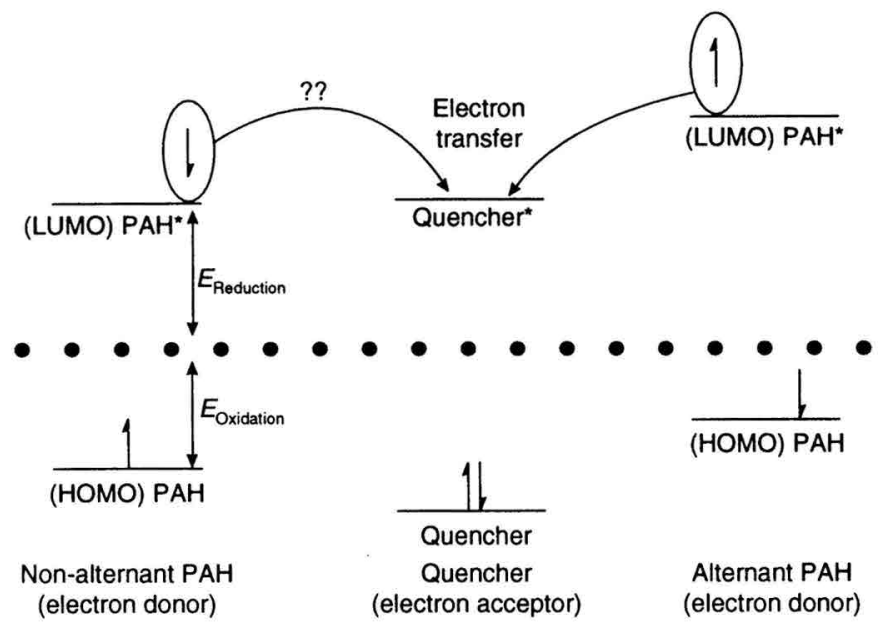

Fig. 4 Simplified molecular orbital diagram indicating favourable conditions for electron transfer between an electron-donor alternant $\mathrm{PAH}$ and an electron-acceptor quenching agent. The quencher's LUMO and non-alternant PAH's LUMO were placed at energies so as to discourage electron transfer (dotted line represents the potential of a reference electrode). Nitromethane quenches only fluorescence emission of alternant PAHs. Intensities of non-alternant PAHs remain unchanged for the most part (a few exceptions are known). More detailed molecular orbital diagrams are given elsewhere. ${ }^{18}$ * Indicates electronically excited state. 
energy $\left({ }^{1} \mathrm{PAH} /{ }^{1} \mathrm{PAH}^{*}\right)-\operatorname{energy}\left({ }^{2} \mathrm{Quencher}-/{ }^{1}\right.$ Quencher $)+$ other term(s)

expected to be more negative in the case of alternant PAHs (see Fig. 4). Assuming that one is in a Marcus-type region, a more favourable electron-transfer process results in larger quenching rate constants, which if comparable to (or larger than) $k_{\text {fluores }}$ would cause a significant decrease in PAH emission intensity. Slow electron transfer reactions (i.e., $\left.k_{\text {quench }} \ll k_{\text {fluores }}\right)$ are not expected to affect fluorescence intensities as the photon is emitted long before electron transfer from the PAH donor to the quencher acceptor can occur (see Fig. 4). A similar argument could be made for electron-donor quenching agents. Here, the quenching agent is more likely to affect fluorescence emission of the nonalternant PAHs (see Fig. 5).

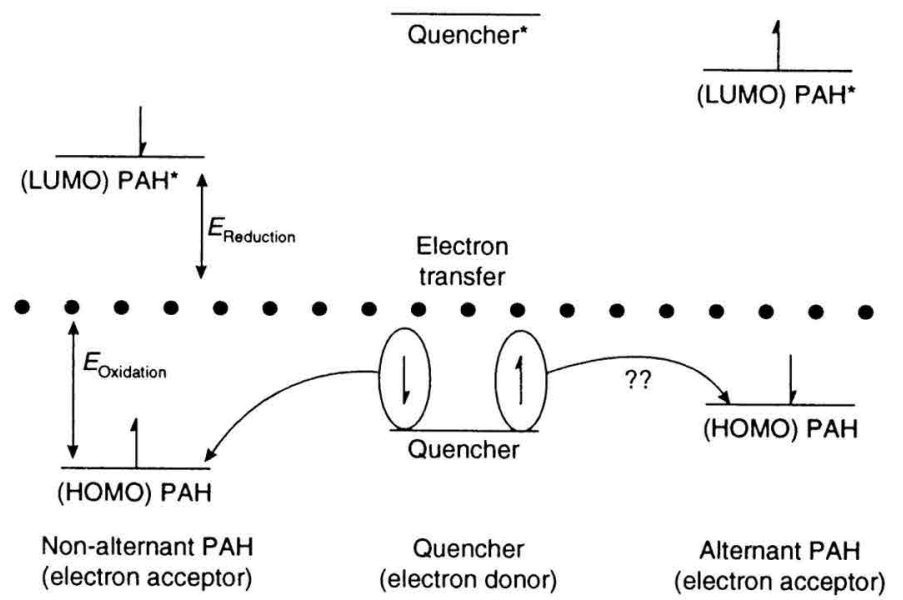

Fig. 5 Simplificd molecular orbital diagram indicating favourable conditions for electron transfer between an electron-acceptor nonalternant PAH and an electron-donor quenching agent, such as 1,2,4trimethoxybenzene. More detailed molecular orbital diagrams are given elsewhere. ${ }^{18 *}$ Indicates electronically excited state.

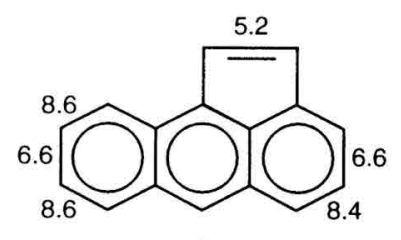

A

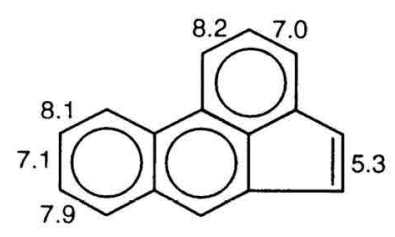

B

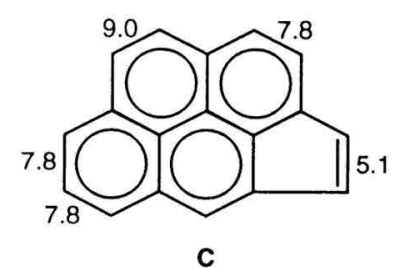

Fig. $6{ }^{1} \mathrm{H}-{ }^{1} \mathrm{H}$ NMR coupling constants for: $\mathrm{A}$, aceanthrylene, $J_{1,2}=$ $5.2 \mathrm{~Hz}, J_{3,4}=6.6 \mathrm{~Hz}, J_{4,5}=8.4 \mathrm{~Hz}, J_{7.8}=8.6 \mathrm{~Hz}, J_{8.9}=6.6 \mathrm{~Hz}$ and $J_{9,10}=8.6 ; \mathrm{B}$, acephenanthrylenc, $J_{1.2}=8.2 \mathrm{~Hz}, J_{2,3}=7.0 \mathrm{~Hz}, J_{4,5}=$ $5.3 \mathrm{~Hz}, J_{7.8}=7.9 \mathrm{~Hz}, J_{8,9}=7.1 \mathrm{~Hz}$ and $J_{9,10}=8.1 \mathrm{~Hz}$; and C, cyclopenta $[c d]$ pyrene, $J_{1.2}=7.8 \mathrm{~Hz}, J_{3.4}=5.1 \mathrm{~Hz}, J_{6,7}=7.8 \mathrm{~Hz}, J_{7,8}$ $=7.8 \mathrm{~Hz}$ and $J_{9.10}=9.0 \mathrm{~Hz} .^{24.33 .34}$
Examination of the quenching data and the molecular orbital diagrams in Figs. 4 and 5 led us to believe that this particular subclass of PAHs, the acenaphthylene derivatives, have HOMO-LUMO energies and energy separations similar to those for the alternant PAHs. Earlier we hypothesized that the peculiar behaviour of the acenaphthylene derivatives might be attributed to the fixed double bond in the cyclopenta ring, which is unlike previously examined non-alternant $\mathrm{PAH}$ subclasses, e.g., fluorenoids and fluoranthenoids. ${ }^{15-21}$ Available NMR data support the conclusions regarding the fixed double bond in the five-membered ring of the various acenaphthylene derivatives. Fig. 6 indicates the coupling constants betwen adjacent protons in aceanthrylene, acephenanthrylene and cyclopenta $[c d]$ pyrene. The coupling constants of the protons at the five-membered ring are significantly smaller than the other coupling constants, and their values $(5.1-5.3 \mathrm{~Hz})$ are outside the region in which vicinal coupling constants of aromatic protons are found (6.6-9.0 $\mathrm{Hz}$ ). A coupling constant of approximately $5 \mathrm{~Hz}$ is normal for protons at the double bond of a cyclopentene derivative, and Fig. 6 illustrates the alkenic rather than aromatic character of the double bond in the five-membered ring of these acenaphthylene-type PAHs. ${ }^{24,32-34}$ The nitromethane selective quenching data involving the acephenanthrylene derivatives indicate that the double bond remains olefinic in nature in the electronic excited state.

This work was supported in part by grants from the National Science Foundation (Grant No. CTS-8922485) and by the University of North Texas Research Council. This work was also supported by a National Science Foundation Doctoral Research Fellowship and an American Chemical Society, Division of Analytical Chemistry, Full-Year Fellowship sponsored by Glaxo, awarded to S. A. Tucher. J. M. Griffin also thanks the UNT Texas Academy of Math and Science (TAMS) for an undergraduate summer research scholarship.

\section{References}

1 Kalman, R. E., J. Basic Eng., 1964, 82, 34.

2 Rutan, S. C., J. Chemometr., 1987, 1, 7.

3 Brown, S. D., Anal. Chim. Acta, 1986, 181, 1.

4 Rutan, S. C., Gerow, D. D., and Hartman, G., Chemometr. Intell. Lab. Syst., 1988, 3, 61.

5 Gampp, H., Maeder, M., Meyer, C. J., and Zuberbühler, A. D., Talanta, 1985, 32, 1133 .

6 Maeder, M., Anal. Chem., 1987, 59, 527.

7 Millican, D. W., and McGown, L. B., Appl. Spectrosc., 1992, 46, 28.

8 Millican, D. W., and McGown, L. B., Anal. Chem., 1989, 61, 580.

9 Millican, D. W., and McGown, L. B., Anal. Chem., 1990, 62, 2242.

10 Vo-Dinh, T., in Modern Fluorescence Spectroscopy, ed. Wehry, L., Plenum Press, New York, 1981, vol. 4, pp. 167-192.

11 Vo-Dinh, T., and Martinex, P. R., Anal. Chim. Acta, 1981, 125 13.

12 Vo-Dinh, T., Gammage, R. B., Hawthorne, A. R., and Thorngate, J. H., Environ. Sci. Technol., 1978, 12, 1297.

13 Blümer, G.-P., and Zander, M., Fresenius' Z. Anal. Chem., 1979, 296, 409.

14 Tucker, S. A., Acree, W. E., Jr., Cho, B. P., Harvey, R. G., and Fetzer, J. C., Appl. Spectrosc., 1991, 45, 1699.

15 Amszi, V. L., Cordero, Y., Smith, B., Tucker, S. A., Acree, W. E., Jr., Yang, C., Abu-Shaqara, E., and Harvey, R. G., Appl. Spectrosc., 1992, 46, 1156.

16 Tucker, S. A., Darmodjo, H., Acree, W. E., Jr., Fetzer, J. C., and Zander, M., Appl. Spectrosc., 1992, 46, 1260.

17 Dreeskamp, H., Koch, E., and Zander, M., Z. Naturforsch., Teil A, 1975, 30, 1311. 
18 Breymann, U., Dreeskamp, H., Koch, E., and Zander, M., Chem. Phys. Lett., 1978, 59, 68.

19 Chen, S. H., Evans, C. E., and McGuffin, V. L., Anal. Chim. Acta, 1991, 246, 65.

20 Tucker, S. A., Acree, W. E., Jr., Fetzer, J. C., Harvey, R. G., Tanga, M. J., Cheng, P.-C., and Scott, L. T., Appl. Spectrosc., 1993, 47, 715.

21 Tucker, S. A., Bates, H. C., Acree, W. E., Jr., and Fetzer, J. C., Appl. Spectrosc., 1993, 47, 1775.

22 Tucker, S. A., Bates, H. C., Amszi, V. L., Acree, W. E., Jr., Harvey. R. G., and Dyker, G., Anal. Chim. Acta, 1993, 278, 269.

23 Ingold, C. K., Structure and Mechanism in Organic Chemistry, Cornell University, Ithaca, 1969, p. 195.

24 Mulder, P. P. J., Bocre, B. B., Baart, A., Cornelisse, J., and and Lugtenburg. J., Recl. Trav. Chim. Pays-Bas, 1993, 112, 22.

25 Parker, C. A., and Barnes, W. J., Analyst, 1957, 82, 606.

26 Holland, J. F., Teets, R. E., Kelly, P. M., and Timnick, A., Anal. Chem., 1977, 49, 706.

27 Yappert, M. C., and Ingle, J. D., Appl. Spectrosc., 1989, 43, 759.
28 Lakowicz, J. R., Principles of Fluorescence Spectroscopy, Plenum Press, New York, 1983.

29 Tucker, S. A., Acree, W. E., Jr., Fetzer, J. C., and Jacob, J., Polycycl. Arom. Cmpds., 1992, 3, 1.

30 Tucker, S. A., Amszi, V. L., and Acree, W. E., Jr., J. Chem. Educ., 1992, 69, A8.

31 Tucker, S. A., Darmodjo, H., Acree, W. E., Jr., Zander, M., Meister, E. C., Tanga, M. J., and Tokita, S., Appl. Spectrosc., 1992, 46, 1630.

32 Sangaiah, R., Gold, A., and Toney, G. E., J. Org. Chem., 1983, 48, 1632.

33 Mulder, P. P. J., Olde Boerrigter, J., Boere, B. B., Zuihof, H., Erkelens, C., Cornelisse, J., and Lugtenburg, J., Recl. Trav. Chim. Pays-Bas, 1993, 112, 287.

34 Jans, A. W. H., Tintel, C., Cornelisse, J., and Lugtenburg, J., Magn. Reson. Chem., 1986, 24, 101.

Paper 3/07113H Received December 1, 1993 Accepted February 24, 1994 